aiming to establish websites to assist those working in the field to conduct research, with supervision and advice 'at a distance'. They founded the organisation Young Investigators in Biological Child and Adolescent Psychiatry, in Germany, with the aim of improving communication and collaboration between young psychiatrists starting out in this area of research. They would like to attract child and adolescent psychiatrists from low- and middle-income countries to join their network, although they acknowledge that the priorities of such psychiatrists may be very different to their own. The increasing availability of e-communication offers an exciting and novel way of promoting research in areas of the world where there are few local experts.

Finally, we consider the specific case of Pakistan, where Drs Khan, Shehzad and Chaudhry consider the difficulties in providing child psychiatry services in a country of 169 million people, of whom $40 \%$ are under the age of 15 years.
There is no specific allocation of funding in Pakistan to this specialty, and no demarcated pathway by which children can be referred for assessment and intervention. Sadly, children are often admitted to adult psychiatric wards, where there is no particular medical or nursing expertise on how to manage their problems. One hope for the future may be a joint paediatric-psychiatric liaison service, but this has yet to take firm root. They emphasise the urgent need for protocols to assist in the management of children. There is also a potential role for mentoring by 'senior volunteers' from the Royal College of Psychiatrists, in a scheme that is attempting to bring senior professionals in the UK into dialogue with psychiatrists (such as those in Pakistan) who would value a mentor. This application of e-communication has the potential to foster the development of a special interest in managing children's mental health among psychiatrists who have trained in adult psychiatry.

\title{
Child and adolescent mental health policy worldwide: an update
}

\section{Jess P. Shatkin MD MPH, ${ }^{1}$ Neaka Balloge BA ${ }^{2}$ and Myron L. Belfer MD MPA ${ }^{3}$}

${ }^{1}$ New York University School of Medicine, NYU Child Study Center, 577 First Avenue, New York, NY 10016, USA, email jess.shatkin@nyumc.org

${ }^{2}$ New York University ${ }^{3}$ Harvard Medical School

E ew countries worldwide maintain policies specifically designed to address the mental health needs of children and adolescents. Yet policies are essential to guide the development of systems of care, training programmes for practitioners, and research endeavours. Without policy, there is no clear pathway for programme development, no specific commitment from government, no expression of governance, no guide to support funding, and no clarification of who exactly is responsible for providing services to children and adolescents. In 2004, we published a report aimed at identifying child and adolescent mental health policies worldwide (Shatkin \& Belfer, 2004). The present review expands upon that report and provides an up-todate assessment of these policies.

As in our prior study, we define a policy as a guide that dictates actions, such as programme development, financing, and access to care. Policy can be formally enacted by law or adopted by decree. Regardless of the level of detail, in all cases policy provides a recognisable document with the presumed force of law. Although there is great discrepancy in the way various countries execute mental health policy in general, the near absence of policies designed specifically to address the mental healthcare needs of children and adolescents, combined with poor execution of those few policies that are currently in existence, is an ever-growing concern. Given that children and adolescents have no political power, policies must be designed to ensure that they are able to access even the most basic mental healthcare.

\section{Background}

The United Nations' 1989 Convention on the Rights of the Child outlines the basic rights to which children are entitled. The right to mental healthcare is a key tenant of the document, and the absence of policies designed to address the mental healthcare needs of children and adolescents stands in direct opposition to the rights established by the Convention. Despite the Convention's ratification by 193 countries, there has been no international enforcement or movement to create mental health policies, leaving most children and adolescents without adequate care.

When considering the lack of child and adolescent mental health policies, one of the most troubling findings is the enormous discrepancy between needed and available resources. Certainly, the mental healthcare available to children and adolescents is far from commensurate with the burden of need. One-half of all lifetime cases of mental illness are now recognised to begin by the age of 14 , and three-quarters by the age of 24, thereby rendering children and adolescents an extremely vulnerable group but with little protection (Kessler et al, 2005). Worldwide prevalence rates of child and adolescent mental health disorders approximate $20 \%$ (one in every five young people), with little variation found among the types of disorders seen across various cultures (US Department of Health and Human Services, 1999; Belfer \& Saxena, 2006). Despite effective treatments, there are long delays, sometimes decades, between the first onset of symptoms and the 
time at which individuals seek and receive treatment, and untreated mental disorders are now recognised commonly to lead to more severe and difficult-to-treat illnesses, along with the development of comorbid disorders (Kessler et al, 2005). Globally, five of the top ten leading causes of disability for children aged 5 years and over are diagnosable mental psychopathologies (Murray \& Lopez, 1996). These findings provide evidence of the large gap that exists between the needed and available services for this extremely vulnerable population.

Clearly, the costs of ignoring the mental healthcare needs of children and adolescents affect both individuals and the whole of society. Children and adolescents who suffer from mental illness are unlikely to reach their full potential when healthcare resources are sparse or unavailable, and sick children are unable to learn and grow to become effective workers. The creation of government policies addressing child and adolescent mental illness is crucial in helping countries first to recognise the burden of illness and then to begin programme development to identify and treat these individuals in a systematic manner.

\section{Method of review}

In an attempt to identify existing mental health policies on a global scale, we performed an extensive review in 2002 (Shatkin \& Belfer, 2004). Given the virtual impossibility of surveying every country worldwide, we employed a literature review methodology, supplemented by contact with consultants and experts in the field at the World Health Organization (WHO) and internationally. Of the 191 countries recognised by the United Nations at that time, we were able to identify only 35 with a mental health policy that might affect children and adolescents. In an effort to categorise each of the policies, we created a four-point classification scheme (Table 1).

The findings from our study provided solid evidence of the gap in child and adolescent mental healthcare policies. Of the 35 countries found to have identifiable mental health policies that may have an effect on children and adolescents, only 14 countries (11 of which are European) qualified for an ' $A$ ' ranking. Consequently, only $7 \%$ of countries worldwide (14 of 191) had any type of clearly articulated policy for dealing with the mental healthcare needs of children and adolescents. Of greatest interest is the fact that the mental health policies

Table 1 Criteria for categorising child and adolescent mental health policies (from Shatkin \& Belfer, 2004)

Category Criteria
A $\quad \begin{aligned} & \text { National policies and/or programme plans that } \\ & \text { recognise the unique mental health and } \\ & \text { developmental problems of children and adolescents } \\ & \text { and clearly enumerate a unifying plan of action }\end{aligned}$
B $\begin{aligned} & \text { National policies and/or programme plans that } \\ & \text { recognise the unique mental health and } \\ & \text { developmental problems of children and adolescents } \\ & \text { but do not enumerate a unifying plan of action }\end{aligned}$
National policies and/or programme plans that
recognise the mental health problems of adults and
are likely to have some direct or indirect beneficial
effect on the mental health of children and
adolescents
No clearly identifiable national policies or programme
plans for mental health

for youths within these countries were all embedded within policies for adults. No single country, out of all we were able to assess, had a stand-alone policy for children and adolescents.

Following up on this research, the WHO aimed to assess further the gap in mental health policies for children and adolescents at an international level. In collaboration with the World Psychiatric Association Presidential Global Program on Child Mental Health and the International Association for Child and Adolescent Psychiatry and Allied Professions, the WHO published the Atlas on child and adolescent mental health resources in 2005 (WHO, 2005). The Atlas project represented a more systematic attempt to identify the gap in resources within child and adolescent mental health policies by employing a more interactive methodology than our prior study (Shatkin \& Belfer, 2004). Data for the Atlas was obtained from countries through the use of a key informant method. Questionnaires requesting both quantitative and qualitative measures of policy performance were sent to select individuals from all WHO member states who were uniquely positioned to provide the relevant information.

\section{Findings}

The Atlas project succeeded in collecting data from 66 nations. While providing relevant and useful information, the Atlas suffered from a low response rate, in large part due to the lack of an identifiable focal point for child and adolescent mental health services, the fragmentation of child and adolescent mental health service systems, and the absence of appropriate mechanisms for gathering data. The inability to gather such valuable data makes policy and service efficacy virtually impossible to calculate.

The findings from the WHO Atlas confirm the absence of adequate epidemiological data relating to child and adolescent mental health disorders in low- and middle-income countries (Belfer \& Nurcombe, 2007). The dearth of such data leads to a poor understanding of the disorders that are most prevalent in these areas and an even more limited understanding of how these disorders are viewed within different cultures. In the vast majority of countries outside of Europe and the Americas, service systems for child and adolescent mental health simply do not exist. Many of these countries rely on hospital or custodial settings for managing mental illness in their youth. As suspected, the Atlas documented that those countries with higher proportions of children in their population (typically poor countries) are also the most lacking in mental health policy for children and adolescents. These findings demonstrate that the gap in mental healthcare resources is greatest in areas that are most deserving of such services.

The number of policies identified by the Atlas project among responders to the survey varies greatly by WHO region. Even these numbers, however, are likely inflated, as identified national policies often were integrated into human rights, social welfare, child protection and education legislation or doctrines. Furthermore, the existence of national health programmes designed to address child and adolescent mental illness was considerably less frequent than the policies themselves, ranging from 6\% in Africa to $83 \%$ in the Western Pacific Region. Consequently, the Atlas data demonstrate that simply having a child and adolescent mental health policy 
in place does not necessarily translate into an identifiable service programme; nor does ratification of the Convention on the Rights of the Child appear to make it more likely that a given country will have a national policy for the provision of child and adolescent mental health services. Lastly, the Atlas project was able to identify an institution or government entity with overall responsibility for child and adolescent mental health services in fewer than one-third of all countries. It is presumed that such services are often supported to varying degrees by ministries of education, social services, and health, but with little communication and coordination.

There are numerous barriers to child and adolescent mental healthcare which remain a significant concern worldwide. Those barriers identified most frequently by the WHO Atlas include lack of resources, transportation difficulties and stigma surrounding mental illness. Of note, stigma associated with the disorders themselves or stigmatisation from one's community was found to be a greater barrier in high-income than in low-income countries. It is suggested that this finding may be due to greater public knowledge of the implications of mental illness in high-income countries, which leads to increased stigma for those diagnosed. By contrast, low-income countries identified transportation difficulties and a lack of available treatment resources as the most significant barriers to receiving care. Additional barriers to care include lack of financial resources and an inability to communicate effectively in the patient's native language.

The Atlas project, coupled with observation of service systems in both higher- and lower-income countries, suggests that a continuum of care is absent. The establishment of such clinical and administrative continuity requires a strong mental health policy. In countries throughout the world, legislatures are far more willing to build buildings than to support home visits or preventive services. The illusion persists that supporting more medicalised care, including the use of pharmaceuticals, will yield greater accountability. However, the evidence suggests that reliance upon in-patient care is not cost-effective and may be counterproductive (Grimes \& Mullin, 2007).

Finally, the WHO Atlas addressed the lack of adequate training programmes and standards for practitioners who provide mental health treatment for children and adolescents (Levav et al, 2004). The Atlas estimates that one-half to twothirds of mental health needs go unmet in most countries. Even in the USA, the world's wealthiest country, child and adolescent psychiatrists are the most under-represented of all medical sub-specialists, with an estimated 6300 practitioners serving a population whose estimated need is over 30000 practitioners (Kim, 2003). As imagined, the situation is far worse in low- and middle-income countries, where, for example, the Atlas was able to identify only ten psychiatrists trained to work with children in the entirety of sub-Saharan Africa outside of South Africa. The Atlas suggests that the lack of trained professionals and stand-alone policies addressing child and adolescent mental illness may be due to a lack of political power, brought about by the absence of children in the political sphere.

The Atlas notes that much of the funding for child and adolescent mental healthcare emanates from temporary and unstable sources rather than from existing government funding. This funding trend was observed in both high- and low-income countries, suggesting that the needs of children and adolescents are often ignored in policy development and financial allotment, due to their invisibility in the political arena. These findings act as further evidence of the need for unique policies to deal with this extremely under-represented population.

\section{Discussion}

Child and adolescent mental health policy discussions are taking place worldwide. Recognising the limited pool of funds allocated to healthcare, professionals involved in the mental health of children and adolescents are becoming stronger advocates and more attuned to the need to compete for funding.

Perhaps the greatest causes of morbidity and mortality among children and adolescents worldwide remain the infectious diseases, yet people who are mentally ill often receive treatment late and do not adhere well to treatments such as antibiotics and other necessary medications. Children and adolescents are no exception. By treating coexisting child and adolescent mental illness and by taking other measures to increase general emotional wellness, the physical health of children and adolescents will also improve. Furthermore, although treatments for children and adolescents with mental illness are continually refined, some of these treatments, primarily medications, carry a significant risk of side-effects, and this evident risk has led to increasing policy discussions. Finally, recent media attention directed to child and adolescent mental health has focused on disease-specific initiatives, such as autism and bipolar disorder, with consequences for national policy - witness the major allocation of money for autism research in the USA brought about through national legislation.

The focus on privatisation of child and adolescent mental health services, along with simultaneous implementation of various forms of managed care and insurance, has led to a troubling trend in policy discussions worldwide. Understandably, yet mistakenly, policies that seek to promote privatised and managed care are being driven, at least in part, by the antipathy of governments to support the presumed unknown or unknowable costs associated with child and adolescent mental health services. These concerns, however, are based on earlier reports of uncontrollable costs. In fact, the available data suggest that improved access to child and adolescent mental health services will be ensured by strong policies in this domain (Grimes \& Mullin, 2007).

As evidenced by our earlier study and the WHO Atlas, no single country worldwide is meeting the documented need for child and adolescent mental health services. Although child and adolescent mental health policies are frequently imperfect and do not necessarily translate into mental health programmes, we continue to suggest that policies are a logical first step towards the recognition of need and subsequent programme development (Belfer, 2007; Belfer et al, 2007).

\section{References}

Belfer, M. L. (2007) Critical review of world policies for mental healthcare for children and adolescents. Current Opinion in Psychiatry, 20, 349-352. 
Belfer, M. L. \& Nurcombe, B. (2007) The epidemiology and burden of child and adolescent mental disorder. In The Mental Health of Children and Adolescents: An Area of Global Neglect (eds H. Remschmidt et al), pp. 27-42. John Wiley.

Belfer, M. L. \& Saxena, S. (2006) WHO child atlas project. Lancet, 367, 551-552.

Belfer, M. L., Remschmidt, H., Nurcombe, B., et al (2007) A global programme for child and adolescent mental health: a challenge in the new millennium. In The Mental Health of Children and Adolescents: An Area of Global Neglect (eds H. Remschmidt et al), pp. 1-11. John Wiley.

Grimes, K. E. \& Mullin, B. (2007) MHSPY: a children's health initiative for maintaining at risk youth in the community. Journal of Behavioral Health Services and Research, 34, 349-351.

Kessler, R. C., Chiu, W. T., Demler, O., et al (2005) Prevalence, severity, and comorbidity of 12-month DSM-IV disorders in the National Comorbidity Survey Replication. Archives of General Psychiatry, 62, 617-627.

Kim, W. J., American Academy of Child and Adolescent Psychiatry Task Force on Workforce Needs (2003) Child and adolescent psychiatry workforce: a critical shortage and national challenge. Academic Psychiatry, 27, 277-282

Levav, I., Jacobson, L., Tsiantis, J., et al (2004) Psychiatric services and training for children and adolescents in Europe: results of a country survey. European Child and Adolescent Psychiatry, 13, 395-401.

Murray, C. J. L. \& Lopez, A. D. (eds) (1996) The Global Burden of Disease. WHO.

Shatkin, J. P. \& Belfer, M. L. (2004) The global absence of mental child and adolescent mental health policy. Child and Adolescent Mental Health, 13, 395-401.

US Department of Health and Human Services (1999) Mental Health: A Report of the Surgeon General. US Department of Health and Human Services, Substance Abuse and Mental Health Services Administration, Center for Mental Health Services, National Institute of Mental Health National Institutes of Health.

WHO (2005) Atlas. Child and Adolescent Mental Health Resources. Global Concerns: Implications for the Future. See http://www.who.int/mental health/resources/Child_ado_atlas.pdf (last accessed August 2008).

\section{Helping each other help children - worldwide research networks in child and adolescent mental health}

\section{Stefan Ehrlich MD ${ }^{1}$ and Paul L. Plener MD²}

${ }^{1}$ Charité - Universitätsmedizin Berlin, Campus Virchow-Klinikum, Klinik für Psychiatrie, Psychosomatik und Psychotherapie des Kindesund Jugendalters, Augustenburger Platz 1, 13353 Berlin, Germany, email stefan.ehrlich@charite.de ${ }^{2}$ Department of Child and Adolescent Psychiatry and Psychotherapy, University of Ulm, Germany

$$
T_{\text {he }}^{\text {he }}
$$
he International Congress of the International Association of Child and Adolescent Psychiatry and Allied Professions (IACAPAP) takes place every other year. It is an opportunity for specialists from around the world to meet, exchange ideas and plan new projects. Since 2006, the Donald J. Cohen (DJC) Travel Fellowship has been an integral part of the Congress. It aims to foster the professional development of 'emerging leaders' in child and adolescent mental health (Adler et al, 2007).

At first glance, child and adolescent psychiatrists from highincome countries would be expected to have very different interests, and very different resources, from those from lowincome countries. Many young German child and adolescent psychiatrists, for example, do biological research using multichannel electroencephalography (EEG), magnetic resonance imaging (MRI), and genetic or cell biology assays. Those from low-income countries might be more interested in such things as the epidemiology of certain disorders in their region, the prevalence of particular conditions, such as child abuse, or the efficacy of community-based mental health programmes.

In Germany, the profession of child and adolescent psychiatry is rather young but well established, the treatment is covered by health insurance, advanced technological tools are accessible, and the medical research infrastructure is complex enough to support some highly specialised projects. In a low-income country there may be only a handful of child and adolescent psychiatrists nationally, the field is often not held in high esteem either by the public or by government, and funding and legislative support for child mental health are correspondingly weak. Health systems generally suffer from low funding and are focused on essential medical needs. Technology such as MRI devices is seldom available. Public health and clinical care take priority over basic research.

When child and adolescent psychiatrists from higher- and lower-income countries meet at a scientific conference, as we and others have under the auspices of the DJC Fellowship, the obvious question therefore is 'Can we help each other?' Which is to say, 'How can we help each other help children?' While forming personal and professional relationships is a good first step, the next is to consider carefully the opportunities for cooperation, and the obstacles.

\section{Tools of research}

If we think not about the specific substance of our research but rather about the tools we use, we see that researchers in higher- and lower-income countries face some similar challenges. Questions arise out of our daily clinical work. We must reframe those into more precise research questions, and devise an adequate research design. This requires access to the existing research literature, as well as resources such as reports from the World Health Organization (WHO). The relatively new journal Child and Adolescent Psychiatry and Mental Health offers free access to peer-reviewed, high-quality, worldwide information on all topics relevant to child and adolescent mental health (Fegert \& Vitiello, 2007). Web forums such as Medscape offer free access to some scientific journals 